Notre Dame Journal of Formal Logic Volume XIX, Number 3, July 1978 NDJFAM

\title{
A NOTE CONCERNING A SOLE SUFFICIENT OPERATOR
}

\author{
J. C. MUZIO
}

Let $\mathrm{E}(k)=\{0,1, \ldots, k-1\}$. In [3] Wesselkamper proves that a particular Markov operator $S$ defined on $\mathrm{E}(k)$ is complete with constants (a function is complete with constants if the set consisting of the function and all the constant functions is complete). In this short note we give an alternative proof of the completeness by showing that Rose's generalized condition disjunction [2] is easily defined using $S$ and the constant functions. This conditioned disjunction is defined by $\left[x, y_{0}, \ldots, y_{k-1}\right]=y_{i}$ if $x=i$ and it was proved in [2] to be complete with constants. (Rose repeats the variable $x$ at the end of the definition to maintain symmetry.)

$$
S \text { is defined by } \operatorname{Sxy} z=\left\{\begin{array}{l}
z, \text { if } x=y \\
x, \text { otherwise. }
\end{array}\right.
$$

We use three sets of definitions, as follows:

D1. $\quad V_{0} x=S 0 S x 001$

$$
V_{j} x=\operatorname{SoSjx} 01, \text { for each } j=1,2, \ldots, k-1 \text {. }
$$

Then $V_{i} x=\left\{\begin{array}{l}1, \text { if } x=i ; \\ 0, \text { otherwise. }\end{array}\right.$

D2. $W_{i} x y=S V_{i} x 1 y$, for each $i, 0 \leqslant i \leqslant k-1$.

Hence $W_{i} x y=\left\{\begin{array}{l}y, \text { if } x=i, \\ 0, \text { otherwise. }\end{array}\right.$

D3. $Q_{1} x y_{0} y_{1}=S W_{0} x y_{0} 0 W_{1} x y_{1}$ $Q_{i+1} x y_{0} y_{1} \ldots y_{i+1}=S Q_{i} x y_{0} \ldots y_{i} 0 W_{i+1} x y_{i+1}$ for each $i=1,2, \ldots, k-2$. Then $\left[x, y_{0}, \ldots, y_{k-1}\right]=Q_{k-1} x y_{0} \ldots y_{k-1}$ so that $S$ is complete with constants. 


\section{REFERENCES}

[1] Markov, A. A., Theory of Mathematical Algorithms, Israel Program for Scientific Translations, Jerusalem (1962), pp. 192-222.

[2] Rose, A., "Conditioned disjunction as a primitive connective for the $m$-valued propositional calculus," Mathematische Annalen, vol. 123 (1951), pp. 76-78.

[3] Wesselkamper, T. C., "A sole sufficient operator," Notre Dame Journal of Formal Logic, vol. XVI (1975), pp. 86-88.

University of Manitoba

Winnipeg, Manitoba, Canada 